\title{
Hierarchical Structuring of NMC111-Cathode Materials in Lithium- Ion Batteries: An In-Depth Study on the Influence of Primary and Secondary Particle Sizes on Electrochemical Performance
}

\author{
Amalia Christina Wagner, Nicole Bohn, Holger Geßwein, Matthias Neumann, Markus Osenberg, \\ André Hilger, Ingo Manke, Volker Schmidt, and Joachim R. Binder*
}

Cite This: ACS Appl. Energy Mater. 2020, 3, 12565-12574

Read Online

ACCESS | Lلll Metrics \& More | 回 Article Recommendations ｜ sl supporting Information

ABSTRACT: Commercially used $\mathrm{LiNi}_{1 / 3} \mathrm{Mn}_{1 / 3} \mathrm{Co}_{1 / 3} \mathrm{O}_{2}$ (NMC111) in lithium-ion batteries mainly consists of a large-grained nonporous active material powder prepared by coprecipitation. However, nanomaterials are known to have extreme influence on gravimetric energy density and rate performance but are not used at the industrial scale because of their reactivity, low tap density, and diminished volumetric energy density. To overcome these problems, the build-up of hierarchically structured active materials and electrodes consisting of microsized secondary particles with a primary particle scale in the nanometer range is preferable. In this paper, the preparation and detailed characterization of porous hierarchically structured active materials with two different median secondary particle sizes, namely, 9 and $37 \mu \mathrm{m}$, and primary particle sizes in the range $300-1200 \mathrm{~nm}$ are presented. Electrochemical investigations by means of rate performance tests show that hierarchically structured electrodes provide higher specific capacities than conventional NMC111, and the cell performance can be tuned by adjustment of processing parameters. In particular, electrodes of coarse granules sintered at $850{ }^{\circ} \mathrm{C}$

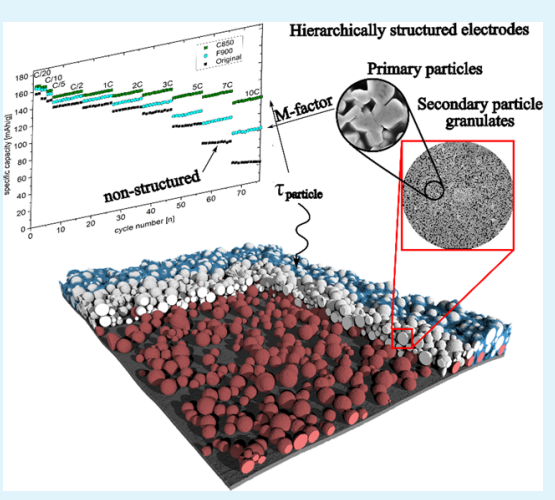
demonstrate more favorable transport parameters because of electrode build-up, that is, the morphology of the system of active material particles in the electrode, and demonstrate superior discharge capacity. Moreover, electrodes of fine granules show an optimal electrochemical performance using NMC powders sintered at $900{ }^{\circ} \mathrm{C}$. For a better understanding of these results, that is, of process-structure-property relationships at both granule and electrode levels, $3 \mathrm{D}$ imaging is performed with a subsequent statistical image analysis. Doing so, geometrical microstructure characteristics such as constrictivity quantifying the strength of bottleneck effects and descriptors for the lengths of shortest transportation paths are computed, such as the mean number of particles, which have to be passed, when going from a particle through the active material to the aluminum foil. The latter one is at lowest for coarsegrained electrodes and seems to be a crucial quantity.

KEYWORDS: hierarchically structured electrodes, process-structure-property relationships, primary particle size effect, secondary particle size effect, porous active material, prediction of effective conductivities, 3D imaging, statistical image analysis

\section{INTRODUCTION}

Energy revolution is nowadays one of the most important topics in scientific research as well as in politics and is essential to reach climate goals. A crucial aspect of energy revolution is to find storage solutions for electrical energy generated exclusively from renewable sources such as wind and solar power, to ensure green mobility free from fossil energy sources. In this respect, lithium-ion batteries (LIBs) are an efficient and green key technology. However, LIBs must ensure battery specifications, which go along with current needs, for example, for locomotion in electric vehicles (EVs) or energy storage. To be comparable to combustion engines, LIBs for automotive applications should reach at least a specific energy density of $350 \mathrm{Wh} / \mathrm{kg}$ and a driving range of more than $500 \mathrm{~km}^{1}$ as well as charging times within less than an hour. At the moment, these goals are not reached yet for pure EVs because cathode materials limit the application because of their smaller amount of cyclable lithium and their restricting transport properties. ${ }^{2,3}$ One way to diminish the gap between theoretical and practical capacity is the alteration of the morphology of active materials and reduction of transport lengths for ions and electrons as already pursued by nanostructuring, which can improve energy densities and rate capability effectively through shortening diffusion paths and improving electronic conductivity., 4 Furthermore, it is known that the mechanical stability of

Received: October 8, 2020

Accepted: November 30, 2020

Published: December 11, 2020 
cathode active materials improves with decreasing primary particle sizes and that nanosized active material particles are less susceptible to crack formation than their bulk, macrosized counterparts. ${ }^{6}$ It is not only the primary particle size that is decisive but also the microstructure of the corresponding secondary particles. For example, the fracture behavior of dense single NCM111 secondary particles consisting of submicron primary particles is dominated by intergranular fracture and independent of the secondary particle size. ${ }^{7}$ Despite all advantages, nanomaterials are more complicated to handle because of their small particle sizes and high surface areas, which provoke side reactions, ${ }^{5}$ agglomeration, and low tap density. ${ }^{8}$ This, in turn, results in capacity fade and low volumetric energy density. ${ }^{9,10}$

To overcome these problems, hierarchically structured materials, where the active material consists of microparticles built up by nanoparticles as subunits, can be used. ${ }^{11}$ For example, Dreizler et al. reported on the benefits of hierarchically structured NMC111 active materials and electrodes compared to nonporous, bulk NMC111. Hierarchically structured NMC111 resulted in higher specific capacities during long-term electrochemical load cycling and showed a different aging behavior. While a nonporous, bulk active material showed considerable crack formation after 8000 cycles, nanostructured powders of hierarchically structured electrodes did not show cracking but exfoliation as the mode of failure. $^{12}$ Nevertheless, to the best of our knowledge, hierarchically structured materials are neither well understood nor investigated in detail or used at the industrial scale in comparison to their bulk counterparts. Furthermore, there are few investigations regarding the influence of size effects in terms of primary and secondary particle sizes of the active material on both the electrochemical performance of hierarchically structured electrodes and the relationships between the morphology of the open porous active materials ${ }^{13}$ and the electrode build-up. As NMC is one of the commonly used active material classes in portable devices and applied in the automobile sector, ${ }^{14}$ we pursue the approach of hierarchical structuring of the NMC111 active material and the investigation of process-structure-property relationships by means of experimental methods and statistical image analysis. Focused ion beam electron scanning microscopy (FIB-SEM) and 3D imaging by synchrotron tomography are suitable tools to investigate the active material's structure and electrode morphology in detail. ${ }^{15,16}$ The aim is to identify morphological differences in the active material and electrode build-up and to correlate them with processing parameters and with the cell performance as this was done for different battery materials in the literature. ${ }^{17-19}$ To do so, geometrical microstructure characteristics, which are known to be important for effective transport properties, such as the volume fraction $\varepsilon$, constrictivity $\beta$, and mean geodesic tortuosity $\tau^{20}$ are computed, for both analyzing the morphology of individual active material particles and the system of active material particles in the electrode. Doing so, the M-factor that is defined as the ratio between effective and intrinsic conductivity is predicted for the individual active material particles and quantifies the influence of nanostructuring on effective transport. Additionally, we introduce a particle-based tortuosity, which seems to be a crucial quantity characterizing the system of active material particles in the electrode. In the following, the target-oriented preparation and characterization of hierarchically structured active materials and electrodes are described. Moreover, the results of the performed electrochemical investigations are given followed by a detailed discussion of morphological effects of active materials and electrodes, which have been determined by statistical image analysis.

\section{EXPERIMENTAL SECTION}

NMC111 from Toda Kogyo Corp. was suspended in deionized water with Darvan $821 \mathrm{~A}$ dispersant and ground in an agitator bead mill (LabStar LS1, Netzsch) with $0.2 \mathrm{~mm} \mathrm{ZrO}_{2}$ beads at $3000 \mathrm{rpm}$. The grinding process was monitored by laser diffraction to determine particle size distribution and mean particle size $d_{50,3}$. As the mean particle size reached a value of around $220 \mathrm{~nm}$, the suspension was split and was spray-dried in a MobileMinor spray dryer from GEA at two different wheel speeds, namely, 39,000 and 12,000 rpm to obtain two different secondary particle sizes. The fractions were sintered at 850,900 , and $950{ }^{\circ} \mathrm{C}$ for $5 \mathrm{~h}$ in air $(5 \mathrm{~L} / \mathrm{min})$ for adjustment of the primary particle size and porosity. The sintered granule powders were characterized as follows: The secondary particle size was determined via laser diffraction (Horiba LA950, Retsch Technology) and the surface area through $\mathrm{N}_{2}$ adsorption with evaluation by BrunauerEmmett-Teller (BET) theory (Gemini VII 2390, Micromeritics), and the granule porosity and pore size distribution was measured by mercury intrusion porosimetry (MIP) (CEI Pascal 1.05, Thermo Electron). The granule porosity $\left(P_{g}\right)$ was calculated from the total pore volume and theoretical density. ${ }^{12}$ FE-SEM investigation (Supra 55, Zeiss) in combination with image analysis (imageJ Software) was used for morphological inspection and estimation of primary particle size distribution, while primary particle sizes are based on Feret diameters. X-ray diffraction (XRD) measurements of the ground powder as well as the spray-dried and sintered materials were performed at a Siemens D5000 diffractometer in reflection mode with $\mathrm{CuK} \alpha$ radiation $\left(\lambda_{1}=1.5406 \AA, \lambda_{2}=1.54443 \AA\right)$, and Rietveld refinement was performed with a TOPAS software package. To check the overall composition after material processing, inductively coupled plasma-optical emission spectrometry (ICP-OES) measurements (iCAP 7600 Duo, Thermo Fisher Scientific) were performed.

Cathodes for electrochemical testing were prepared by mixing PvdF binder (KYNAR, Powerflex LBG-1), active materials, and carbon black (Timcal, C65) in a 1:8:1 ratio in NMP and doctor blading the slurry at $200 \mu \mathrm{m}$ gap height on an aluminum current collector. The foils were dried overnight at $80{ }^{\circ} \mathrm{C}$ and were processed to half cells without calendering. Active material loadings of $6-7 \mathrm{mg} /$ $\mathrm{cm}^{2}$ were obtained. The electrode layer porosities $\left(P_{\mathrm{E}}\right)$ were calculated from the layer mass, thickness, and theoretical layer density $\left(3.62 \mathrm{~g} / \mathrm{cm}^{3}\right)$. The layer thickness was acquired by measurement with a thickness gauge at 10 spots on each electrode layer.

Swagelok-type cells were built from the as-prepared cathode layers, a Whatman GF/C separator, a Li-anode, and $80 \mu \mathrm{L}$ LP30 electrolyte (1 $\mathrm{M} \mathrm{LiPF}_{6}, \mathrm{v} / \mathrm{v}:$ 1:1 DMC:EC) from Sigma-Aldrich. For electrochemical testing, a BT2000 battery cycler from Arbin Instruments was used. Galvanostatic cycling at discharge rates of C/20, C/10, C/5, C/ $2,1 \mathrm{C}, 2 \mathrm{C}, 3 \mathrm{C}, 5 \mathrm{C}, 7 \mathrm{C}$, and $10 \mathrm{C}$ with $1 \mathrm{C}$ corresponding to $183 \mathrm{mAh} /$ $\mathrm{g}$ was performed in the voltage range between 4.3 and $3.0 \mathrm{~V}$, whereas the charging rate was retained at $\mathrm{C} / 2$ from $1 \mathrm{C}$ on.

As in our previous publication, ${ }^{21}$ experimental characterization of the microstructure of the considered NMC111 electrodes is complemented by statistical image analysis. In the present paper, the results are based on 3D image data on two different length scales.

The inner structure within individual secondary particles was investigated by means of FIB-SEM tomography using a ZEISS Crossbeam 350. For milling, a gallium gun was used with an energy of $30 \mathrm{keV}$ and a current of $3 \mathrm{nA}$. The serial cross sections were scanned with an electron energy of $1 \mathrm{keV}$ and a pixel size of $10 \mathrm{~nm}$. Data drift correction, image brightness homogenization, and thresholding were performed using Fiji resulting in classified 3D data sets with an isotropic voxel size of $10 \mathrm{~nm}$. 


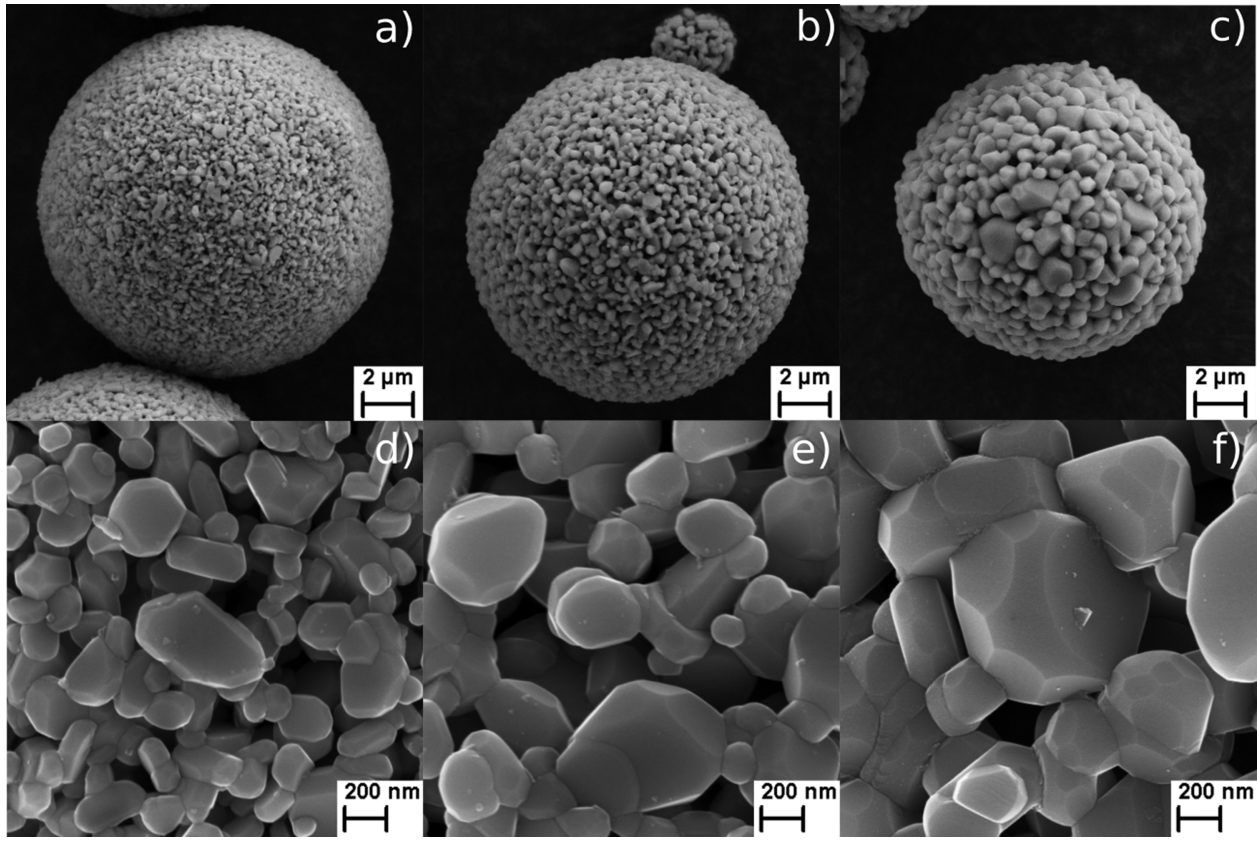

Figure 1. Scanning electron microscopy (SEM) micrographs of differently manufactured microsized active material particles: (a) F850, (b) F900, and (c) F950. In (d) - (f), corresponding primary particles are visualized at higher magnification.

To investigate the structure of the ensemble of secondary particles in the electrode, synchrotron tomography ${ }^{22,23}$ was performed at DESY (P05) and at BESSY II (BAMline). The electrodes were measured with a photon energy of 25 and $30 \mathrm{keV}$ for thin and thick electrodes, respectively, resulting in a combined phase and absorption based contrast. A Paganin filter was applied to transform and enhance the phase contrast of the porous binder-additive phase. For the segmentation, that is, the classification of the aluminum foil of the electrode, the binder-additive-pore phase, and the active material, the trainable Weka segmentation ${ }^{24,25}$ was used. A random forest classifier was manually trained on $0.5 \%$ of the measured raw data.

For image data of both length scales, we computed the volume fraction $\varepsilon$ and experimentally nonaccessible microstructure characteristics of the active material such as the constrictivity $\beta$ and mean geodesic tortuosity $\tau_{\text {geod }}{ }^{20,26,27}$ quantifying the strength of bottleneck effects and the length of shortest transportation paths, respectively. Additionally to $\tau_{\text {geod }}$, we compute for each active material particle the minimum number of particles, which have to be passed, when going from this particle through the active material to the aluminum foil, see the sketch in Figure S5 of Supporting Information Part B. This number is then normalized by the minimum distance of the particle center to the aluminum foil. The corresponding mean value can be considered as a particle-based tortuosity, which we denote by $\tau_{\text {particle. }}$. Constrictivity is defined by $\beta=r_{\min }^{2} / r_{\max }^{2}$, where $r_{\min }$ is a geometrically defined radius of the typical bottleneck and $r_{\max }$ is the maximum radius $r$ such that $50 \%$ of the considered phase can be covered by spheres of radius $r$ being completely contained in this phase. For prediction of the $\mathrm{M}$-factor, that is, the ratio of effective over intrinsic conductivity, we use an empirically derived prediction formula. ${ }^{20}$ The latter one was used to predict the M-factor of primary particles within individual secondary particles. The main results of statistical image analysis are shown and discussed in Section 3.3. Additional descriptions are given in Supporting Information Part B.

\section{RESULTS AND DISCUSSION}

3.1. Active Material Characterization. After spray drying, spherical granules with mean secondary particle sizes of around $9 \mu \mathrm{m}$ for the fine powders and $37 \mu \mathrm{m}$ for the coarse powders (Figure 1 and Figure S1 in Supporting Information Part A) are obtained. Mean granule sizes coincide for the sintering temperatures of 850,900 , and $950{ }^{\circ} \mathrm{C}$ for fine and coarse granules, and identical trends, namely, increasing primary particle sizes and decreasing surface areas and granule porosity with increasing sintering temperature, are observed because of densification and grain growth (Table 1). The

Table 1. Powder Characteristics of the Original Starting Material and Fine and Coarse Granules ${ }^{a}$

$\begin{array}{cccccccc}\text { sample } & \text { F850 } & \text { F900 } & \text { F950 } & \text { C850 } & \text { C900 } & \text { C950 } & \text { original } \\ \begin{array}{c}\text { SPsize } d_{50,3} \\ {[\mu \mathrm{m}]}\end{array} & 8.3 & 8.8 & 9.0 & 37.3 & 37.1 & 37.1 & 8.4 \\ \begin{array}{c}\text { PPsize } d_{50,3} \\ {[\mathrm{~nm}]}\end{array} & 357 & 471 & 944 & 412 & 594 & 1199 & 765 \\ \begin{array}{c}A_{\mathrm{BET}}\left[\mathrm{m}^{2} / \mathrm{g}\right] \\ P_{\mathrm{G}}[\%]\end{array} & 4.3 & 2.6 & 0.5 & 3.4 & 1.6 & 0.7 & 0.4 \\ \begin{array}{c}\text { modes of pore } \\ \text { sizes [nm }]\end{array} & 113 & 158 & 180 & 43 & 31 & 18 & \\ \end{array}$

${ }^{a} \mathrm{SP}=$ secondary particles, $\mathrm{PP}=$ primary particles, $\mathrm{SP}$ sizes are obtained by laser diffraction, PP sizes were computed by means of image analysis, $A_{\mathrm{BET}}=$ specific surface area determined by means of BET Theory, and $P_{\mathrm{g}}=$ granule porosity deduced from MIP.

powders are denoted as ' $\mathrm{F}$ ' for fine and ' $\mathrm{C}$ ' for coarse-sintered granules and designated as original for the nonstructured NMC111 reference material from now on. The sintering temperature will be given as an additional annotation, for example, F850, for fine granules sintered at $850{ }^{\circ} \mathrm{C}$. An extreme leap in the primary particle size is observed between 900 and $950{ }^{\circ} \mathrm{C}$ for both granule sizes. It can be explained with grain growth mechanisms, which originate from volume diffusion and diffusion along grain boundaries. ${ }^{28}$ In contrast, the original material occurs as irregular and partially broken aggregate particles with a mean secondary particle size of 8.4 $\mu \mathrm{m}$, comparable to the fine ones. The corresponding powder cross section is shown in Figure S2 of Supporting Information Part A. The original material shows a mean primary particle size of $765 \mathrm{~nm}$, a small surface area of $0.4 \mathrm{~m}^{2} / \mathrm{g}$, and a more or 

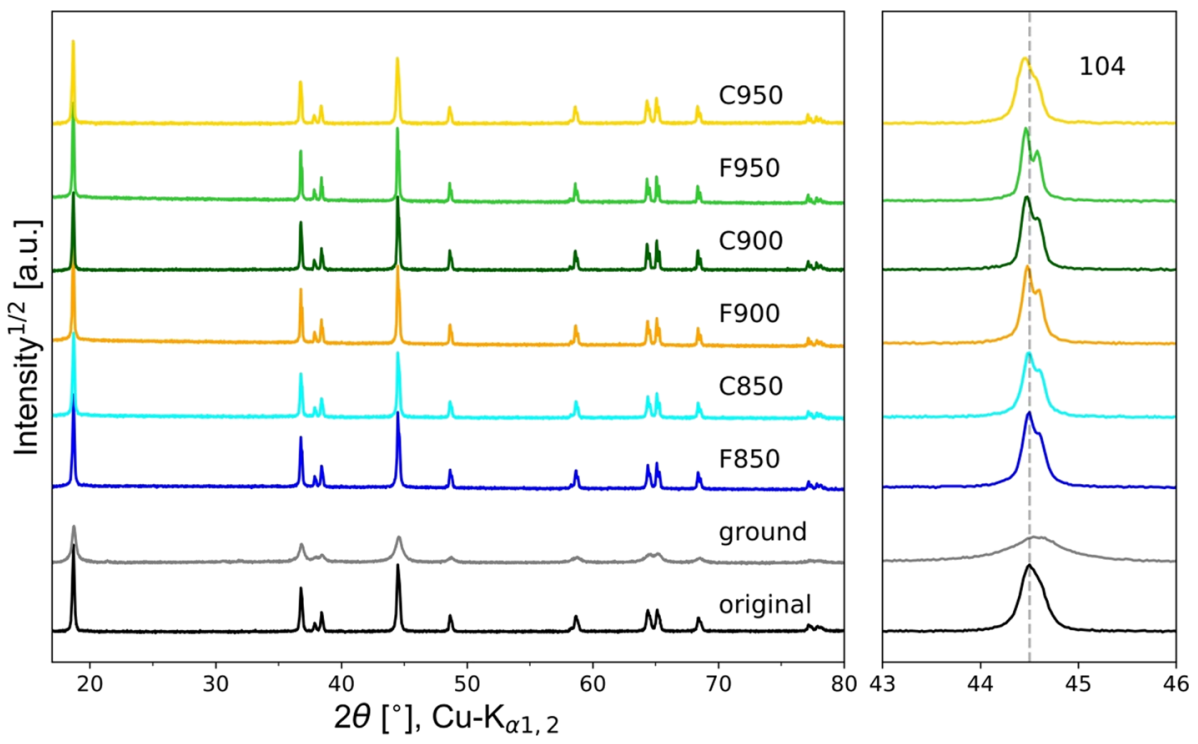

Figure 2. XRD patterns for the original powder, primary particles ground in water, and the hierarchically structured samples F850-F950 and C850C950 after sintering.

a)

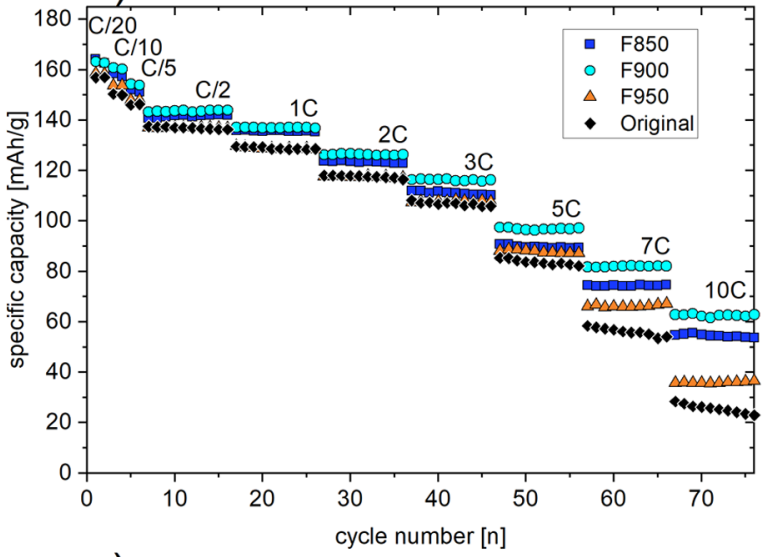

c)

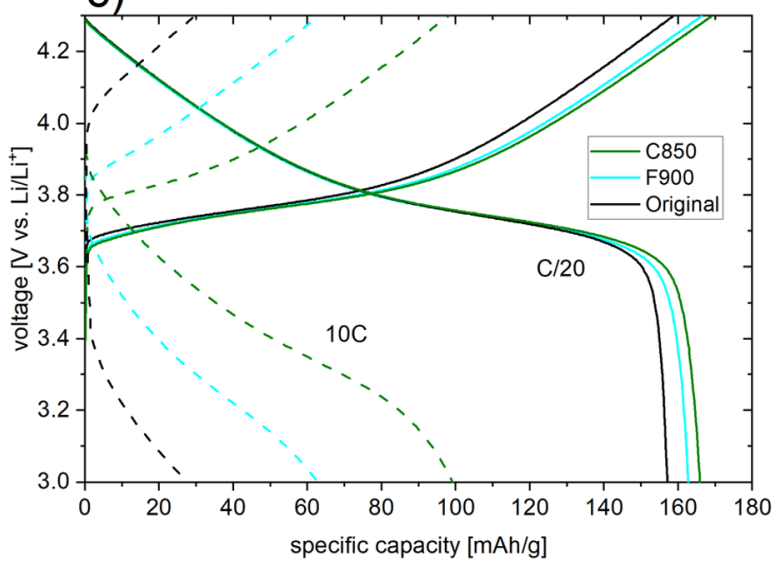

b)

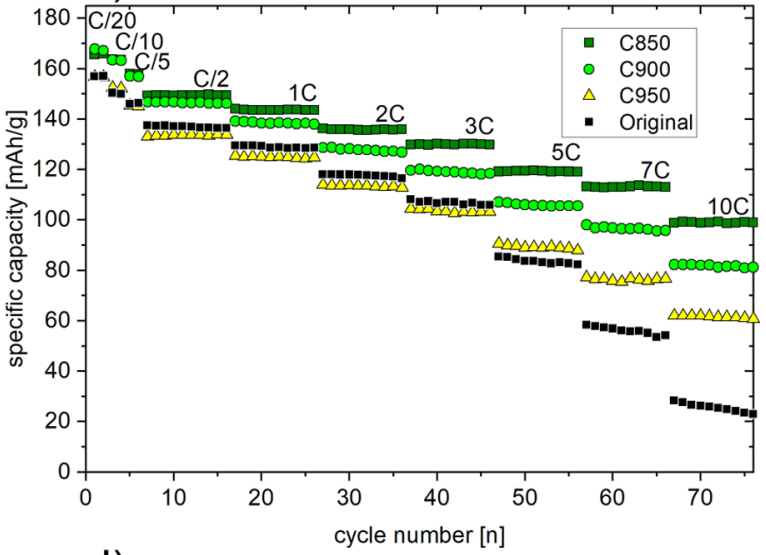

d)

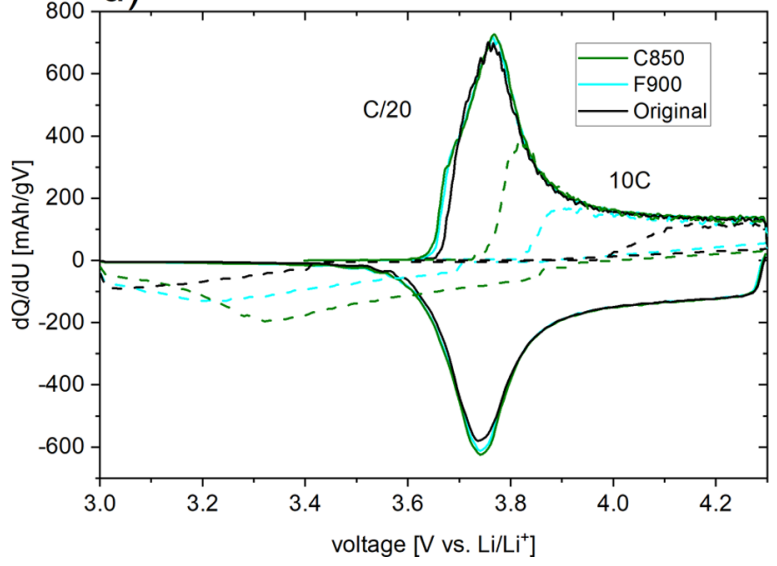

Figure 3. Rate performance tests for hierarchically structured electrodes of (a) F850-F950 and (b) C850-C950 in the voltage range of 3.0-4.3 V. (c) Voltage-capacity plots of C850, F900, and the original reference material at C/20 (solid) and 10C (dashed) discharging rate. (d) dQ/dU-plots of C850, F900, and the original reference material at C/20 (solid) and 10C (dashed).

less dense inner structure with no open porosity. ${ }^{12}$ The mean primary particle size of the original powder lies in between the sizes of correspondingly structured powders sintered at 900 and $950{ }^{\circ} \mathrm{C}$, respectively (Table 1 ).
For fine and coarse granules sintered at 850, 900, and 950 ${ }^{\circ} \mathrm{C}$, porosities of 46 and $43 \%, 38$ and $31 \%$, and 20 and $18 \%$, respectively, were obtained, which result from particle coarsening and sintering. Likewise, modes of pore sizes are increasing because of the coarsening of solid and pore phases. 
A good accessibility of the pore phase is ensured because of macropores for all granule powders and the low surface tension of generally used organic electrolytes. Noteworthy are the differences in the primary particle sizes for fine and coarse granules despite identical temperature treatment. Coarse granules lead to slightly higher primary particle sizes and lower porosities than fine granules after sintering at the same temperature, which indicates a slightly deviating sintering behavior, even though the same ground powder was used for the production of these cathode materials.

The XRD patterns shown in Figure 2 of the considered fine and coarse granulated powders provide insights into material integrity and purity after annealing. All patterns represent NMC111, which crystallizes in space group $R 3 \bar{m}$ in the $\alpha$ $\mathrm{NaFeO}_{2}$ structure type. No impurity phases are found after grinding and sintering. The ground primary particles give the typical peak broadening according to crystallite sizes in the submicrometer range. From Rietveld refinement, crystallites with $22 \mathrm{~nm}$ size were obtained, whereas the nanostructured granules are well-crystallized powders and show no broadening at all. Lattice parameters are given in Table S1 of Supporting Information Part A. C/a-ratios are above 4.97 for all granules, which indicates a well-ordered layered structure ${ }^{28}$ before grinding and after sintering. The c/a-ratio is slightly increasing for each granule size with temperature. Slight differences in lattice parameters of fine and coarse granules point out that there are small differences in the atomic scale like exchange of $\mathrm{Li}^{+}$and $\mathrm{Ni}^{2+}$ on their positions. The synthetic route is known to have an influence on the c/a-ratio. ${ }^{29}$ Lattice parameters are nevertheless in good agreement with literature data ${ }^{30}$ for slightly overlithiated NMC111. ICP-OES measurements complementarily proved compositional integrity of the considered sintered fine and coarse granules with a slight excess of lithium of up to $2 \%$. The original starting material contains a surplus of $5 \%$ and is in good accordance with granulated materials. Note that the analysis of the granulated active material by ICP-OES and the prior dissolution of the active material do not disclose differentiation of $\mathrm{Li}$ belonging to $\mathrm{Li}_{2} \mathrm{CO}_{3}$ on the surface. In general, compositional integrity of the material is maintained during spray granulation so that the electrochemical performance will be ascribed to morphological characteristics of the active material.

3.2. Electrochemical Investigations. Figure $3 a, b$ displays the results of C-rate-dependent cycling tests of hierarchically structured electrodes with fine and coarse granules and their nonstructured counterpart to investigate differences in performance caused by morphological characteristics. Discharge capacities were tested between $\mathrm{C} / 20$ and $10 \mathrm{C}$ and give the typical grading of electrochemical performance depending on the C-rate caused by kinetic restrictions of the active material and the electrodes. It is obvious that improved discharge capacities over all C-rates can be obtained for the low-temperature sintered hierarchically structured electrodes F850, F900 and C850, C900 compared to the nonstructured active material. Depending on the granule size, the optimal sintering temperatures seem to be different for fine and coarse granules, so that electrodes with $9 \mu \mathrm{m}$ secondary particles perform best for all C-rates, when sintered at $900{ }^{\circ} \mathrm{C}$, whereas for coarse secondary particles, a sintering temperature of 850 ${ }^{\circ} \mathrm{C}$ seems to work better. In addition, performances of electrodes with fine granules are much closer to each other than those of the electrodes with coarse granules.
F900 delivers mean discharge capacities of around 161 $\mathrm{mAh} / \mathrm{g}$ at $\mathrm{C} / 20,100 \mathrm{mAh} / \mathrm{g}$ at $5 \mathrm{C}$, and $60 \mathrm{mAh} / \mathrm{g}$ at $10 \mathrm{C}$ with good cycling stability for consecutive cycles at the same discharge rate. C850 provides 164,120 , and $98 \mathrm{mAh} / \mathrm{g}$, whereas the original nonporous material shows capacities of 158,85 , and $28 \mathrm{mAh} / \mathrm{g}$ for corresponding C-rates. F950 and C950 electrodes perform more or less comparable or slightly worse than the nonstructured reference electrode up to $3 \mathrm{C}$ but they outperform it at even higher rates. Qualitative trends are known from nanostructured agglomerated powders ${ }^{31}$ but seem also to hold true for hierarchically structured active materials combining properties of macro and nanoparticles. As the ratecontrolling step for fast charge and discharge is the solid-state diffusion of $\mathrm{Li}^{+}$-ions in NMC111 because of its low diffusion coefficient, ${ }^{32}$ a reduction of the diffusion length is known to speed up the diffusion rate proportionally to $L^{2} / D$, where $L$ is the diffusion length and $D$ is the diffusion coefficient. ${ }^{1}$ This effect is clearly visible at medium and high cycling rates for low-temperature sintered hierarchically structured electrodes compared to nonstructured electrodes or electrodes of hightemperature sintered granules. Furthermore, the combination of small primary particle sizes, high surface areas, and porosities contributes essentially to discharge capacities because the porosity has an effect on the ionic conductivity of $\mathrm{Li}^{+}$inside the granules and the extraction of lithium out of it. A comparable effect is known from microcracks in the active material, which allow the electrolyte to penetrate the material more effectively. ${ }^{33}$ In this regard, the effect of inner porosity for F950 and C950 is seen at high rates compared to the original material, though the larger primary particle sizes limit the diffusion. The diffusion lengths are drastically reduced for F850/F900 and C850/C900, and a good electrolyte penetration is given, so that solid-state diffusion of $\mathrm{Li}^{+}$-ions is not limiting anymore, and the electron transport inside the granule active material plays a more significant role. The Coulombic efficiencies of the first and second cycles are given in Table S2 of Supporting Information Part A. These values prove that the hierarchically structured electrodes consume less lithium during the formation process and that they perform more efficient during the performance test compared to the original nonstructured electrode.

In Figure 3c, voltage-capacity plots of C850, F900, and the nonstructured original material at $\mathrm{C} / 20$ and $10 \mathrm{C}$ are given to illustrate the differences in overall performance. In general, especially at the beginning, a different course of the charging curves is observed for the hierarchically structured electrodes compared to the nonstructured ones, so that the potential window is used more efficiently. During discharge at low rates, the potentials for the structured samples follow more or less the same trends with a prolonged discharging time leading to more reinserted lithium for hierarchically structured samples. At the highest current rate (10C), the discharge curves of cells with a nanostructured cathode material are clearly shifted to higher voltages and show a shallower decline, which indicates the kinetic benefit of this electrode structure. First of all, differences can be explained by increasing conductivity with granule sizes. Furthermore, $\mathrm{Li}^{+}$-ions ensue a surface conduction mechanism, and electrons tend to pass through the granules. ${ }^{34}$

From the $\mathrm{dQ} / \mathrm{dU}$-plots at $\mathrm{C} / 20$ (Figure $3 \mathrm{~d}$ ), the typical redox response of NMC111 as the superposition of the $\mathrm{Ni}^{2+}$ / $\mathrm{Ni}^{4+}$ - and $\mathrm{Co}^{2+} / \mathrm{Co}^{3+}$-redox reactions during delithiation and lithiation is seen. The curves for hierarchically structured electrodes deviate from the usual course during lithiation 


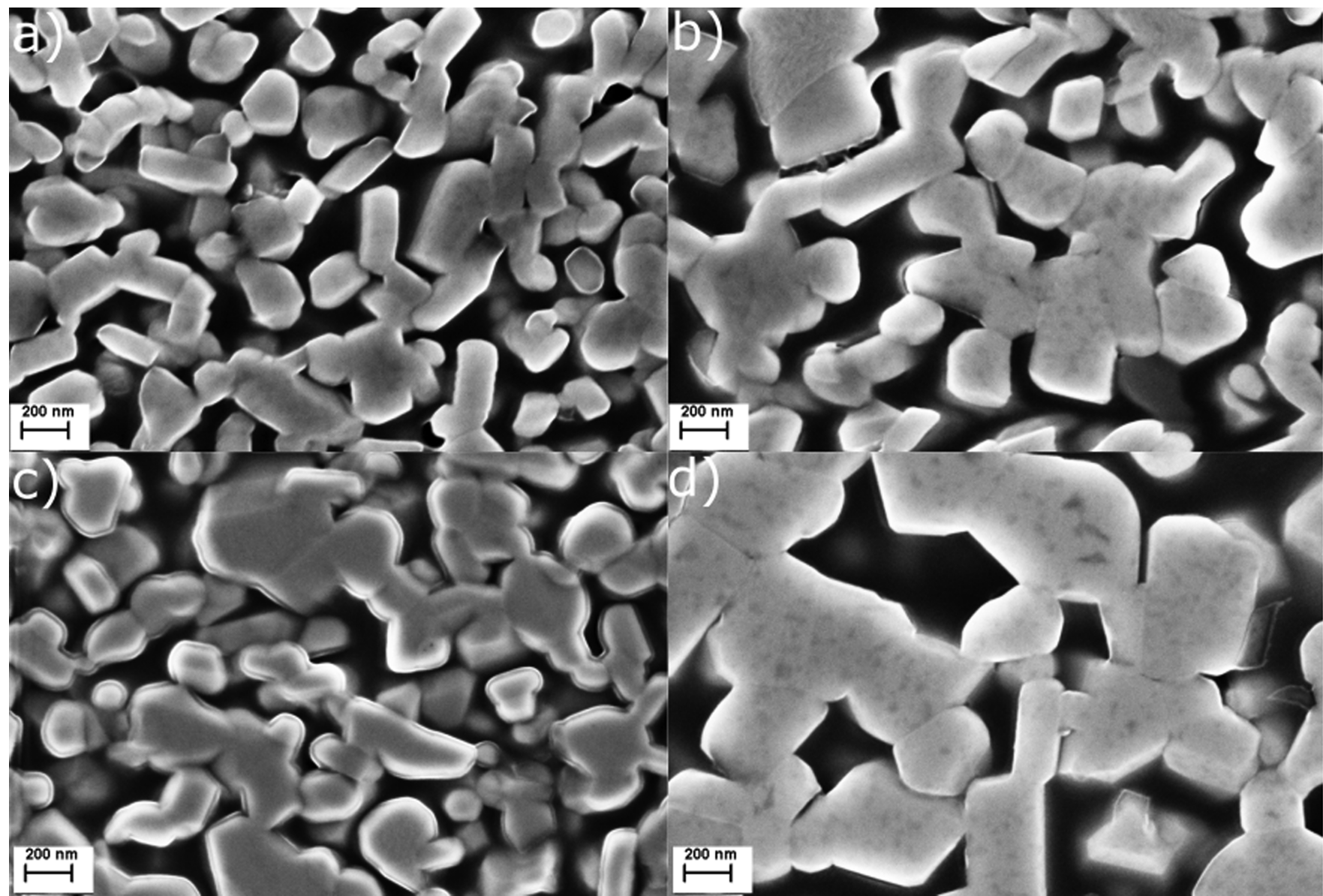

Figure 4. Cross sections of secondary particles sintered at (a) $850{ }^{\circ} \mathrm{C}$ (F850) and (b) $900{ }^{\circ} \mathrm{C}$ (F900) and corresponding coarse granules sintered at $850{ }^{\circ} \mathrm{C}(\mathrm{C} 850)$ in $(\mathrm{c})$ and $900{ }^{\circ} \mathrm{C}(\mathrm{C} 900)$ in (d).

especially for small voltages $(3.65-3.75 \mathrm{~V})$ by a small shoulder. The mentioned shape varies with morphological characteristics by varying temperature treatment. A slight change of the charging curve up to $3.8 \mathrm{~V}$ in the first cycle had been reported by $\mathrm{Li}$ et al. and was assumed to stem from the preparation method and the true chemical composition for their materials. ${ }^{18}$ In our case, it might also stem from the surface layers of granules as the particle surface could deviate in composition compared to the inner part of the granules because of the manufacturing process. However, the significantly larger surface area alone could lead to a different course of the potential curve. Going to 10C, strong polarization effects are seen as shifts between redox-peaks and a grading of polarization is recognized, comparable to the observed performance with the nonstructured electrodes having the largest polarization. A more detailed description of the observed structure-property relationship requires a more detailed analysis of the geometrical microstructure characteristics.

3.3. Morphological Analysis of Individual Nanostructured Active Material Particles. To evaluate morphological differences of fine and coarse granule active materials and the electrode build-up, FIB-SEM imaging as well as synchrotron tomography was performed. Statistical image analysis of F850/F900- and C850/C900-granule powders was evaluated because their performance differs significantly despite the same sintering conditions. For individual active material particles, the $\mathrm{M}$-factors, defined as the ratio of effective over intrinsic conductivity, were predicted by means of geometrical microstructure characteristics. ${ }^{20}$ Figure 4 visualizes cross sections of the granules F850, F900, and their coarse counterparts. Cross sections of F950 and C950 are displayed in Figure S4 of Supporting Information Part A.

Doing so, a M-factor of 0.46 for F900 and 0.20 for F850 was obtained. For coarse granules, the M-factor increases to 0.47 for $\mathrm{C} 900$ and 0.38 for C850. The effect of sintering temperature on the formation of the granule microstructure and thus on effective electronic conductivity is more pronounced for fine than for coarse granules because the $\mathrm{M}$ factor is more than doubled when increasing the temperature by $50{ }^{\circ} \mathrm{C}$. As can be seen for F850, the granule structure shown in Figure $4 \mathrm{a}$ exhibits small primary particle sizes and with that smaller and less areas of contact between primary particles, while F900 shows a strong coarsening with formation of larger contact areas. The structure variation for the fine granules is in good agreement with the formerly mentioned increase of the $\mathrm{M}$-factor. For coarse granules, the increase of effective conductivity because of the increase of temperature amounts just to $24 \%$, whereas the M-factor in general is larger for 850 ${ }^{\circ} \mathrm{C}$. The observed changes in the M-factor for F850 and F900 are in good agreement with our electrochemical observation that F900 performs better than F850, although the primary particles are larger. Despite the same trend in the M-factor for coarse granules, C850 and C900 do not follow the trend of electrochemical performance precisely as C850 performs superior to C900. Predictions of the M-factor for the pore 
Table 2. Mean Values and Standard Deviations of Layer Properties of Hierarchically Structured Electrodes (F850-F950 and C850-C950) and the Nonstructured Reference Electrode (Original) ${ }^{a}$

\begin{tabular}{|c|c|c|c|c|c|c|c|}
\hline electrode & F850 & F900 & F950 & $\mathrm{C} 850$ & C900 & $\mathrm{C} 950$ & original \\
\hline loading $\left[\mathrm{mg} / \mathrm{cm}^{2}\right]$ & $6.3 \pm 0.6$ & $6.2 \pm 0.5$ & $6.5 \pm 0.3$ & $5.9 \pm 0.8$ & $6.3 \pm 0.7$ & $6.5 \pm 0.6$ & $6.6 \pm 0.2$ \\
\hline layer thickness $[\mu \mathrm{m}]$ & $76 \pm 5$ & $71 \pm 6$ & $66 \pm 6$ & $86 \pm 5$ & $79 \pm 7$ & $75 \pm 4$ & $50 \pm 4$ \\
\hline$\varepsilon(\mathrm{NMC})$ without $P_{\mathrm{g}}[\%]$ & $18 \pm 3$ & $19 \pm 2$ & $21 \pm 2$ & $15 \pm 2$ & $17 \pm 2$ & $18 \pm 2$ & $28 \pm 2$ \\
\hline$\varepsilon(\mathrm{NMC})$ including $P_{\mathrm{g}}[\%]$ & $32 \pm 5$ & $30 \pm 3$ & $26 \pm 3$ & $27 \pm 4$ & $24 \pm 3$ & $22 \pm 2$ & - \\
\hline electrode porosity $P_{\mathrm{E}, \mathrm{g}}$ with $P_{\mathrm{g}}[\%]$ & $71 \pm 3$ & $69 \pm 3$ & $66 \pm 3$ & $63 \pm 5$ & $73 \pm 3$ & $70 \pm 3$ & $54 \pm 3$ \\
\hline electrode porosity $P_{\mathrm{E}}[\%]$ & $57 \pm 5$ & $58 \pm 5$ & $61 \pm 4$ & $51 \pm 7$ & $65 \pm 4$ & $66 \pm 3$ & $54 \pm 3$ \\
\hline$\varepsilon(\operatorname{PvdF})[\%]$ & $11 \pm 2$ & $12 \pm 1$ & $13 \pm 1$ & $9 \pm 1$ & $11 \pm 1$ & $12 \pm 1$ & $18 \pm 1$ \\
\hline
\end{tabular}

${ }^{a} P_{\mathrm{g}}=$ granule porosity, $P_{\mathrm{E}, \mathrm{g}}=$ electrode porosity including granule porosity, and $P_{\mathrm{E}}=$ electrode porosity without granule porosity

a)

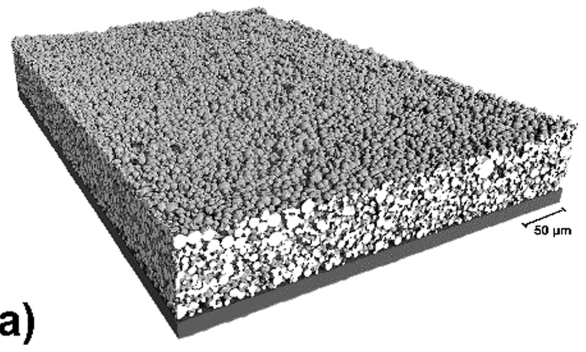

b)

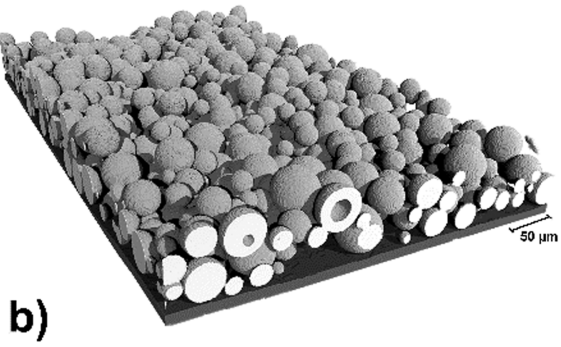

c)

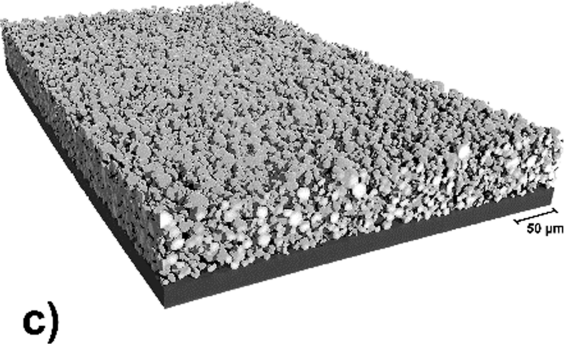

Figure 5. 3D images of hierarchically structured electrode layers containing (a) F900 granules, (b) C900 granules, and (c) the nonstructured NMC111 original active material.

phase, which can be interpreted as the ratio of effective over intrinsic diffusivity, ${ }^{35}$ behave contrarily to the M-factor of the solid phase. In particular, we obtained the values of $0.33,0.10$, 0.17 , and 0.10 for F850, F900, C850, and C900, respectively. This suggests that in contrast to the fine granules, the differences in the predicted effective conductivity are less important for $\mathrm{C} 850$ and $\mathrm{C} 900$ but rather the $\mathrm{Li}^{+}$diffusion into the active material and thus the different primary particle size.

In Figure S3, a summary of the rate performance tests in dependence on granule sizes is displayed and outlines the superior performance of C850 compared to F900. Why the coarse granules-independent of the inner structure of the active materials-show a better performance than the fine granules is not clear from intrinsic properties of the material. To elucidate the observed differences with respect to performance, the electrode build-up, that is, the morphology of the system of active material particles in the electrode will be analyzed and discussed in the next section.

3.4. Morphological Analysis of the System of Active Material Particles in the Electrode. In this section, we evaluate the effect of secondary particle sizes and active material porosity on the morphology of the electrode. For this purpose, geometrical characteristics such as the layer thickness, porosity, and volume fractions of the constituents were experimentally determined, and statistical analysis of 3D image data obtained by synchrotron tomography was performed. The latter one enables for the computation of experimentally nonaccessible microstructure characteristics. The experimentally computed characteristics are summarized in Table 2.

A decreasing trend in the layer thickness for electrodes with fine and coarse secondary particles is observed with increasing sintering temperature. For the layers with fine granules, slightly smaller layer thicknesses are obtained because of the more regular packing of F850-F950. The electrode porosity $P_{\mathrm{E}}$ is between 57 and $61 \%$ for fine and between 51 and $66 \%$ for coarse granules, respectively, with an increasing trend in sintering temperature. In general, these are typical values for uncalendered hierarchically structured electrodes, and fine and coarse electrodes individually show more or less constant electrode porosities for all sintering temperatures, which is a quality feature of the considered layers. Accordingly, $P_{\mathrm{E}, \mathrm{g}}$, which is the electrode porosity including granule porosity, is even higher for fine and coarse hierarchically structured electrodes and shows a decreasing trend going from F850 to F950 and from C900 to C950 because of the varying sintering temperature and inner porosity. Nevertheless, the ratio of layer thickness to granule size is quite different for fine and coarse electrode layers. Layers containing fine granules accumulate around eight granules over their height and are multigranule layers, while layers of coarse granules contain just around one to two stacked granules. Differences prevail because of the arrangement of fine and coarse granules, secondary particle size distribution, and the worse packing density of coarse secondary particles. The nonstructured NMC111 layer exhibits a porosity $P_{\mathrm{E}}$ of $54 \%$ and gives a slightly denser electrode packing because of the irregular particle shapes, which can fill the space more efficiently. Regarding electrode porosity, it is common that a reduced porosity can improve electrochemical performance as known from calendaring. ${ }^{19}$ Note that the porosity of the reference layer with the nonstructured active material is comparable to F850 while its performance is comparable to that of F950. This observation strongly suggests that microstructural effects beyond porosity are crucial for the performance of the considered electrodes.

In Figure 5, the reconstructed 3D images of the hierarchically structured fine and coarse electrodes F900 and C900 as well as the nonstructured original electrode are visualized. In Figure 5b, the larger secondary particle size of C900 can be recognized compared to F900 and the nonstructured electrode in Figure 5a,c, which display comparable secondary particle sizes and a visually similar electrode build-up.

Based on tomographic image data representing the morphology of the system of active material particles in the 
electrode, we compute further microstructure characteristics by statistical image analysis, which are experimentally not accessible. The results are shown in Table 3.

Table 3. Mean Values and Standard Deviations of Transport-Relevant Parameters Computed from 3D Image Analysis

\begin{tabular}{lcccc} 
sample & $\varepsilon$ & $\tau_{\text {geod }}$ & $\tau_{\text {particle }}\left[\mu \mathrm{m}^{-1}\right]$ & $\beta$ \\
F850 & $0.31 \pm 0.01$ & $1.29 \pm 0.02$ & $0.18 \pm 0.01$ & $0.08 \pm 0.01$ \\
F900 & $0.34 \pm 0.01$ & $1.28 \pm 0.05$ & $0.18 \pm 0.01$ & $0.12 \pm 0.03$ \\
F950 & $0.30 \pm 0.01$ & $1.33 \pm 0.03$ & $0.20 \pm 0.01$ & $0.09 \pm 0.02$ \\
C850 & $0.24 \pm 0.02$ & $1.15 \pm 0.16$ & $0.01 \pm 0.00$ & $0.16 \pm 0.21$ \\
C900 & $0.35 \pm 0.03$ & $1.21 \pm 0.10$ & $0.03 \pm 0.00$ & $0.05 \pm 0.01$ \\
C950 & $0.28 \pm 0.03$ & $1.54 \pm 0.69$ & $0.02 \pm 0.01$ & $0.11 \pm 0.14$ \\
original & $0.33 \pm 0.01$ & $1.24 \pm 0.02$ & $0.14 \pm 0.00$ & $0.20 \pm 0.00$ \\
\hline
\end{tabular}

Note that for the computation of transport-relevant microstructure characteristics concerning the system of active material particles, the inner porosity of granules is not taken into account, and the spatial arrangement of the additivebinder phase is not considered. The volume fractions computed by means of image analysis are in reasonable accordance with those obtained from experiments, see Table 2 . The experimentally determined volume fractions of the active material increase with the temperature because of sintering. Deviations between the experiment and image analysis regarding the volume fractions occur for C900 and C950. Note that the electrodes with coarse active material particles are relatively thin compared to the size of their active material particles, see Figure 5b. Moreover, it is not clear a priori which voxels, being classified as void, are pore voxels of the electrode and which of them are outside of the electrode. This influences the results with respect to the volume fractions of C850, C900, and C950 significantly. The values for mean geodesic tortuosity $\tau_{\text {geod }}$ are between 1.29 and 1.33 for electrodes with fine secondary particles, whereas for hierarchically structured electrodes with coarse secondary particles, these values are between 1.15 and 1.54. The constrictivities of electrodes with coarse granules amount to $0.05-0.16$ and to $0.08-0.12$ for electrodes with fine granules. In general, constrictivities and mean geodesic tortuosities close to 1 means that there are no bottlenecks and transportation paths going directly through the structure. The obtained results regarding the volume fraction, mean geodesic tortuosity, and constrictivity do not allow for an explanation of the experimental results with respect to the performance of the electrodes, see Figure 3. Furthermore, we computed $\tau_{\text {particle }}$ as defined in Section 2. The obtained mean values are given in Table 3, while the histograms representing the distribution corresponding to $\tau_{\text {particle }}$ are shown in Figure S6 of Supporting Information Part B. Here, one can observe that $\tau_{\text {particle }}$ is much smaller for C850-C950 (0.01-0.03) compared to F850-F950 (0.18-0.20), which means that the conduction paths have to pass fewer resistances between different active material particles in the case of coarse granules. Our analysis suggests that this might be the dominant factor influencing the performance. In particular, the electrode that performs best (C850) has the lowest value of $\tau_{\text {particle. }}$. Moreover, the performance of nanostructured electrodes with fine granules is better than the one of electrodes with nonstructured active material despite a lower value of $\tau_{\text {particle }}$ (0.14) for the original electrode considered in the present paper. This means that the effect of nanostructuring is more pronounced, but provided that nanostructuring is performed, $\tau_{\text {particle }}$ seems to be a crucial quantity.

\section{CONCLUSIONS}

We have presented a simple procedure of hierarchically structuring active materials by means of grinding and spray drying for the active material NMC111 and have shown how the variation of process parameters regarding the sintering temperature and spray parameter can influence the morphology and the size of individual active material particles, the morphology of the system of active material particles in the electrode, and the electrochemical performance of the electrode. In general, it was observed that granules sintered at 850 and $900{ }^{\circ} \mathrm{C}$ show the best electrochemical performance, but differences prevail because of the granule size and electrode morphology. For increasing sintering temperature, the primary particle sizes are increasing, which is the major limit for ion diffusion inside the active material. For structured porous NMC111 granules with small primary particle sizes in the range between 350 and $550 \mathrm{~nm}$, ion diffusion is not limiting anymore, so that the dominating transport mechanism is the electron conductivity. The latter one is influenced by the granule structure and areas of contact between primary particles. For the morphology of individual secondary particles, the $\mathrm{M}$-factor, which describes the influence of the morphology on effective conductivity, is a reliable descriptor of granule coarsening and grain growth. Its value doubles going from F850 to F900. The same trend is observed in the electrochemical performance of F900, which performs superior to F850. The worst performance among all structured active materials is observed at $950{ }^{\circ} \mathrm{C}$, which matches almost the performance for the original reference material because of the large primary particle sizes. In general, it can be said that there are two opposed effects of ionic and electronic conductivity on the secondary particles scale, which are influenced by the geometry of the underlying active material structure. While larger primary particles reduce the effective Li-ion conductivity, well-developed contact areas between primary particles support electron conduction. Hierarchically structured electrodes consisting of coarse secondary particles show improved performance compared to fine ones, which is a combination of two effects. First, the geometrically predicted effective conductivity predicted by the M-factors is larger for C850 and C900 compared to F850 and F900. Second, the layer properties are more optimal. While fine granule powders allow for a slightly denser packing, the coarse ones show more favorable transport characteristics in terms of lower values of $\tau_{\text {particle. }}$ This is in accordance with the measured electrochemical performance of $\mathrm{C} 850$ as the best-performing electrode and the superior performance compared to the electrodes with fine granules. Altogether, the presented results show the potential of hierarchical structuring of battery materials as well as the possibility to tailor the microstructure and thus the electrochemical properties of the materials via the underlying process parameters. The processes used for the synthesis are scalable and are suitable for the production of hierarchically structured NCM111 materials on a production scale. In general, the reproducibility of the postprocessing and the spray drying process is ensured at constant process parameters. However, a subsequent conditioning of the battery materials would not be economical, so that the structuring has to be done already during the production process of the NCM materials. A further disadvantage of the presented structured 
NCM materials is the high total porosity of the corresponding electrodes of up to $75 \%$. In further work, it could be shown that by calendering the electrodes, a densification of up to $40 \%$ total porosity is possible without losing the advantageous properties of the hierarchically structured materials.

\section{ASSOCIATED CONTENT}

\section{SI Supporting Information}

The Supporting Information is available free of charge at https://pubs.acs.org/doi/10.1021/acsaem.0c02494.

SEM micrographs, data tables: lattice parameters and tap density, performance tests, table with 1 st and 2 nd cycle Coulombic efficiency, sketch visualizing $\tau_{\text {particle, }}$ and data from statistic image analysis for $\tau_{\text {particle }}$ (PDF)

\section{AUTHOR INFORMATION}

\section{Corresponding Author}

Joachim R. Binder - Institute for Applied Materials (IAMESS), Karlsruhe Institute of Technology, 76344 EggensteinLeopoldshafen, Germany; Email: joachim.binder@kit.edu

\section{Authors}

Amalia Christina Wagner - Institute for Applied Materials (IAM-ESS), Karlsruhe Institute of Technology, 76344 Eggenstein-Leopoldshafen, Germany

Nicole Bohn - Institute for Applied Materials (IAM-ESS), Karlsruhe Institute of Technology, 76344 EggensteinLeopoldshafen, Germany

Holger Geßwein - Institute for Applied Materials (IAMESS), Karlsruhe Institute of Technology, 76344 EggensteinLeopoldshafen, Germany

Matthias Neumann - Institute of Stochastics, Ulm University, 89069 Ulm, Germany

Markus Osenberg - Department of Materials Science and Technology, TU Berlin, 10623 Berlin, Germany

André Hilger - Department of Materials Science and Technology, TU Berlin, 10623 Berlin, Germany

Ingo Manke - Department of Materials Science and Technology, TU Berlin, 10623 Berlin, Germany; (1) orcid.org/0000-0001-9795-5345

Volker Schmidt - Institute of Stochastics, Ulm University, 89069 Ulm, Germany

Complete contact information is available at: https://pubs.acs.org/10.1021/acsaem.0c02494

\section{Notes}

The authors declare no competing financial interest.

\section{ACKNOWLEDGMENTS}

This work has been supported by the German Federal Ministry for Economic Affairs and Energy (BMWi) and granted through Project Management Jülich (03ET6095A, 03ET6095B, and 03ET6095E). Additional funding was provided by the Deutsche Forschungsgemeinschaft (DFG, MA 5039/4-1, project number 357753796). We are grateful to the research center DESY (Hamburg, Germany), a member of the Helmholtz Association HGF, for provisioning of experimental facilities. Parts of this research were carried out at PETRAIII and we would like to thank Fabian Wilde for assistance in using P05. Furthermore, we thank Dr. Thomas Bergfeldt for performing the ICP-OES measurements. This work contrib- utes to the research performed at the Center for Electrochemical Energy Storage (CELEST) Ulm-Karlsruhe.

\section{REFERENCES}

(1) Chen, R.; Zhao, T.; Zhang, X.; Li, L.; Wu, F. Advanced cathode materials for lithium-ion batteries using nanoarchitectonics. Nanoscale Horiz. 2016, 1, 423-444.

(2) Goodenough, J. B.; Park, K.-S. The Li-ion rechargeable battery: A perspective. J. Am. Chem. Soc. 2013, 135, 1167-1176.

(3) Li, J.; Wang, Y.; Wu, J.; Zhao, H.; Liu, H. CNT-embedded $\mathrm{LiMn}_{0.8} \mathrm{Fe}_{0.2} \mathrm{PO}_{4} / \mathrm{C}$ microsphere cathode with high rate capability and cycling stability for lithium ion batteries. J. Alloys Compd. 2018, 731, 864-872.

(4) Chen, J. A review of nanostructured lithium ion battery materials via low temperature synthesis. Recent Pat. Nanotechnol. 2013, 7, 2-12.

(5) Jo, M.; Hong, Y.-S.; Choo, J. Effect of $\mathrm{LiCoO}_{2}$ cathode nanoparticle size on high rate performance for Li-ion batteries. $J$. Electrochem. Soc. 2009, 156, A430-A434.

(6) Chen, D.; Kramer, D.; Mönig, R. Chemomechanical fatigue of $\mathrm{LiMn}_{1.95} \mathrm{Al}_{0.05} \mathrm{O}_{4}$ electrodes for lithium-ion batteries. Electrochim. Acta 2018, 259, 939-948.

(7) Dang, D.; Wang, Y.; Cheng, Y.-T. Communication-Fracture behavior of single $\mathrm{LiNi}_{0.33} \mathrm{Mn}_{0.33} \mathrm{Co}_{0.33} \mathrm{O}_{2}$ particles studied by flat punch indentation. J. Electrochem. Soc. 2019, 166, A2749-A2751.

(8) Wang, Y.; He, P.; Zhou, H. Olivine $\mathrm{LiFePO}_{4}$ : Development and future. Energy Environ. Sci. 2011, 4, 805-817.

(9) Wang, M.; Yang, Y.; Zhang, Y. Synthesis of micro-nano hierarchical structured $\mathrm{LiFePO}_{4} / \mathrm{C}$ composite with both superior high-rate performance and high tap density. Nanoscale 2011, 3, 44344439.

(10) Ye, S.; Lv, J.; Gao, X.; Wu, F.; Song, D. Synthesis and electrochemical properties of $\mathrm{LiMn}_{2} \mathrm{O}_{4}$ spinel phase with nanostructure. Electrochim. Acta 2004, 49, 1623-1628.

(11) Radin, M. D.; Hy, S.; Sina, M.; Fang, C.; Liu, H.; Vinckeviciute, J.; Zhang, M.; Whittingham, M. S.; Meng, Y. S.; van der Ven, A. Narrowing the gap between theoretical and practical capacities in $\mathrm{Li}$ ion layered oxide cathode materials. Adv. Energy Mater. 2017, 7, 1602888.

(12) Dreizler, A. M.; Bohn, N.; Geßwein, H.; Müller, M.; Binder, J. R.; Wagner, N.; Friedrich, K. A. Investigation of the influence of nanostructured $\mathrm{LiNi}_{0.33} \mathrm{Co}_{0.33} \mathrm{Mn}_{0.33} \mathrm{O}_{2}$ lithium-ion battery electrodes on performance and aging. J. Electrochem. Soc. 2018, 165, A273A282.

(13) Lin, B.; Wen, Z.; Gu, Z.; Huang, S. Morphology and electrochemical performance of $\mathrm{Li}\left[\mathrm{Ni}_{1 / 3} \mathrm{Co}_{1 / 3} \mathrm{Mn}_{1 / 3}\right] \mathrm{O}_{2}$ cathode material by a slurry spray drying method. J. Power Sources 2008, 175, $564-569$.

(14) Sakti, A.; Michalek, J. J.; Fuchs, E. R. H.; Whitacre, J. F. A techno-economic analysis and optimization of Li-ion batteries for light-duty passenger vehicle electrification. J. Power Sources 2015, 273, 966-980.

(15) Etiemble, A.; Besnard, N.; Bonnin, A.; Adrien, J.; Douillard, T.; Tran-Van, P.; Gautier, L.; Badot, J.-C.; Maire, E.; Lestriez, B. Multiscale morphological characterization of process induced heterogeneities in blended positive electrodes for lithium-ion batteries. J. Mater. Sci. 2017, 52, 3576-3596.

(16) Cocco, A. P.; Nelson, G. J.; Harris, W. M.; Nakajo, A.; Myles, T. D.; Kiss, A. M.; Lombardo, J. J.; Chiu, W. K. S. Three-dimensional microstructural imaging methods for energy materials. Phys. Chem. Chem. Phys. 2013, 15, 16377-16407.

(17) Neumann, M.; Wagner, A.; Bohn, N.; Osenberg, M.; Hilger, A.; Manke, I.; Binder, J. R.; Schmidt, V. Characterization of hierarchically structured electrodes with different thicknesses by means of experiments and image analysis. Mater. Charact. 2019, 155, 109778.

(18) Ebner, M.; Chung, D.-W.; García, R. E.; Wood, V. Tortuosity anisotropy in lithium-ion battery electrodes. Adv. Energy Mater. 2014, 4, 1301278

(19) Kang, H.; Lim, C.; Li, T.; Fu, Y.; Yan, B.; Houston, N.; de Andrade, V.; de Carlo, F.; Zhu, L. Geometric and electrochemical 
characteristics of $\mathrm{LiNi}_{1 / 3} \mathrm{Mn}_{1 / 3} \mathrm{Co}_{1 / 3} \mathrm{O}_{2}$ electrode with different calendering conditions. Electrochim. Acta 2017, 232, 431-438.

(20) Neumann, M.; Stenzel, O.; Willot, F.; Holzer, L.; Schmidt, V. Quantifying the influence of microstructure on effective conductivity and permeability: Virtual materials testing. Int. J. Solids Struct. 2020, 184, 211-220.

(21) Neumann, M.; Cabiscol, R.; Osenberg, M.; Markötter, H.; Manke, I.; Finke, J. H.; Schmidt, V. Characterization of the 3D microstructure of Ibuprofen tablets by means of synchrotron tomography. J. Microsc. 2019, 274, 102-113.

(22) Banhard, J.. Advanced Tomographic Methods in Materials Research and Engineering; Oxford University Press: Oxford, 2008.

(23) Maire, E.; Withers, P. J. Quantitative X-ray tomography. Int. Mater. Rev. 2013, 59, 1-43.

(24) Arganda-Carreras, I.; Kaynig, V.; Rueden, C.; Eliceiri, K. W.; Schindelin, J.; Cardona, A.; Sebastian Seung, H. Trainable Weka segmentation: A machine learning tool for microscopy pixel classification. Bioinformatics 2017, 33, 2424-2426.

(25) Schindelin, J.; Arganda-Carreras, I.; Frise, E.; Kaynig, V.; Longair, M.; Pietzsch, T.; Preibisch, S.; Rueden, C.; Saalfeld, S.; Schmid, B.; Tinevez, J.-Y.; White, D. J.; Hartenstein, V.; Eliceiri, K.; Tomancak, P.; Cardona, A. Fiji: an open-source platform for biological-image analysis. Nat. Methods 2012, 9, 676-682.

(26) Holzer, L.; Wiedenmann, D.; Münch, B.; Keller, L.; Prestat, M.; Gasser, P.; Robertson, I.; Grobéty, B. The influence of constrictivity on the effective transport properties of porous layers in electrolysis and fuel cells. J. Mater. Sci. 2013, 48, 2934-2952.

(27) Neumann, M.; Hirsch, C.; Staněk, J.; Beneš, V.; Schmidt, V. Estimation of geodesic tortuosity and constrictivity in stationary random closed sets. Scand. J. Statist. 2018, 46, 848-884.

(28) Zhu, J.; Vo, T.; Li, D.; Lu, R.; Kinsinger, N. M.; Xiong, L.; Yan, Y.; Kisailus, D. Crystal growth of $\mathrm{Li}\left[\mathrm{Ni}_{1 / 3} \mathrm{Co}_{1 / 3} \mathrm{Mn}_{1 / 3}\right] \mathrm{O}_{2}$ as a cathode material for high-performance lithium ion batteries. Cryst. Growth Des. 2012, 12, 1118-1123.

(29) Shin, Y.; Choi, W.; Hong, Y.; Yoon, S.; Ryu, K.; Chang, S. Investigation on the microscopic features of layered oxide $\mathrm{Li}$ $\left[\mathrm{Ni}_{1 / 3} \mathrm{Co}_{1 / 3} \mathrm{Mn}_{1 / 3}\right] \mathrm{O}_{2}$ and their influences on the cathode properties. Solid State Ionics 2006, 177, 515-521.

(30) Zhang, X.; Jiang, W. J.; Mauger, A.; Gendron, F.; Julien, C. M. Minimization of the cation mixing in $\mathrm{Li}_{1+\mathrm{x}}(\mathrm{NMC})_{1-\mathrm{x}} \mathrm{O}_{2}$ as cathode material. J. Power Sources 2010, 195, 1292-1301.

(31) Li, D.-C.; Muta, T.; Zhang, L.-Q.; Yoshio, M.; Noguchi, H. Effect of synthesis method on the electrochemical performance of $\mathrm{LiNi}_{1 / 3} \mathrm{Mn}_{1 / 3} \mathrm{Co}_{1 / 3} \mathrm{O}_{2}$. J. Power Sources 2004, 132, 150-155.

(32) Li, X.; Liu, J.; Banis, M. N.; Lushington, A.; Li, R.; Cai, M.; Sun, $\mathrm{X}$. Atomic layer deposition of solid-state electrolyte coated cathode materials with superior high-voltage cycling behavior for lithium ion battery application. Energy Environ. Sci. 2014, 7, 768-778.

(33) Amin, R.; Chiang, Y.-M. Characterization of electronic and ionic transport in $\mathrm{Li}_{1-\mathrm{x}} \mathrm{Ni}_{0.33} \mathrm{Mn}_{0.33} \mathrm{Co}_{0.33} \mathrm{O}_{2}$ (NMC333) and $\mathrm{Li}_{1-\mathrm{x}} \mathrm{Ni}$ ${ }_{0.50} \mathrm{Mn}_{0.20} \mathrm{Co}_{0.30} \mathrm{O}_{2}$ (NMC523) as a function of $\mathrm{Li}$ content. $J$. Electrochem. Soc. 2016, 163, A1512-A1517.

(34) Burkhardt, S.; Friedrich, M. S.; Eckhardt, J. K.; Wagner, A. C.; Bohn, N.; Binder, J. R.; Chen, L.; Elm, M. T.; Janek, J.; Klar, P. J. Charge transport in single NCM cathode active material particles for lithium-ion batteries studied under well-defined contact conditions. ACS Energy Lett. 2019, 2117-2123.

(35) Neumann, M.; Furat, O.; Hlushkou, D.; Tallarek, U.; Holzer, L.; Schmidt, V. On microstructure-property relationships derived by virtual materials testing with an emphasis on effective conductivity. In Simulation science: First International Workshop, SimScience 2017, Göttingen, Germany, April 27-28, 2017 : revised selected papers; Baum, M.; Brenner, G.; Grabowski, J.; Hanschke, T.; Hartmann, S.; Schöbel, A., Eds.; Communications in computer and information science 889; Springer: Cham, Switzerland, 2018; pp. 145-158. 